\title{
Trivium
}

Revue franco-allemande de sciences humaines et sociales - Deutsch-französische Zeitschrift für Geistesund Sozialwissenschaften

$10 \mid 2012$

Lisibilité

\section{Hepatische Empathie: Die Affinität des Inkommensurablen nach Aby Warburg}

\section{Georges Didi-Huberman}

Traducteur : Markus Sedlaczek

\section{CpenEdition}

\section{Journals}

Édition électronique

URL : http://journals.openedition.org/trivium/4179

DOI : $10.4000 /$ trivium.4179

ISSN : 1963-1820

Éditeur

Les éditions de la Maison des sciences de l'Homme

Référence électronique

Georges Didi-Huberman, « Hepatische Empathie: Die Affinität des Inkommensurablen nach Aby Warburg », Trivium [Online], 10 | 2012, online erschienen am 30 März 2012, abgerufen am 07

September 2020. URL : http://journals.openedition.org/trivium/4179; DOI : https://doi.org/10.4000/ trivium. 4179

Ce document a été généré automatiquement le 7 septembre 2020.

\section{cc)}

Les contenus des la revue Trivium sont mis à disposition selon les termes de la Licence Creative Commons Attribution - Pas d'Utilisation Commerciale - Pas de Modification 4.0 International. 


\title{
Hepatische Empathie: Die Affinität des Inkommensurablen nach Aby Warburg
}

\author{
Georges Didi-Huberman \\ Traduction : Markus Sedlaczek
}

\section{NOTE DE L'ÉDITEUR}

Wir danken Georges Didi-Huberman für die freundliche Genehmigung, diesen Artikel zu übersetzen.

Der Artikel, unveröffentlicht in vorliegender Form, bildet die Grundlage des ersten Teils von George Didi-Hubermans Monographie Atlas ou le gai savoir inquièt. L'oeil de l'histoire t. 3, Paris: Ed. de Minuit, 2011.

1 Der Begriff der Empathie bzw. Einfühlung hat, ebenso wie der des Pathos, im gängigen Diskurs der Kunsthistoriker und Kunstkritiker bekanntlich eine eher schlechte Presse. Gleichwohl gilt es festzuhalten, dass die Einfühlung* zu den Grundelementen der Kunstgeschichte als einer modernen Disziplin gehört, und zwar in ihrer sogenannt "formalistischen" wie in ihrer »ikonologischen" Version. Die Sache ist bereits bei Heinrich Wölfflin bekannt, insbesondere in seinem Text Prolegomena zu einer Psychologie der Architektur ${ }^{1}$ aus dem Jahre 1886. Bei Aby Warburg, dessen ikonologischer Forschungsansatz allzu oft auf Fragen der »Inhalte«, der archivalischen »Quellen« oder der literarischen »Symbole« reduziert wird, scheint das weniger evident zu sein. Ich möchte im Folgenden, in den Grenzen des vorliegenden Aufsatzes, einfach in Erinnerung rufen, welche Bedeutung Fragen der Empathie bei Warburg besaßen, und zwar sowohl im Umfeld der ersten von ihm publizierten Arbeit - seiner Dissertation über die mythologischen Bilder Botticellis - als auch im Umfeld seiner letzten, unvollendeten Unternehmung, des Bilderatlas Mnemosyne. 
2 Wir wissen, dass Warburg nach zwei aufeinanderfolgenden Durchsichten der Druckfahnen $\mathrm{zu}$ seiner Dissertation im Jahre 1892 plötzlich beschloss, eine „Vorbemerkung« hinzuzufügen, die in nur fünfzehn Zeilen das bemerkenswerte theoretische Programm skizzierte, das bereits in seinen Forschungen zu den »Quellen« Botticellis impliziert war und dem er sein ganzes Leben lang verpflichtet bleiben sollte. ${ }^{2}$ Dieser kurze Text erscheint also, in aller Bescheidenheit, als die einzige Absichtserklärung - die einzige epistemologische Stellungnahme - des jungen Kunsthistorikers am Rande dessen, was er gerne seine "verworrene Gelehrsamkeit « ${ }^{3}$ nannte. Um ihn besser zu verstehen, müssen wir jedoch noch drei weitere Elemente berücksichtigen: Zum einen wollte Warburg seinen Versuch mit einer theoretischen Schlussfolgerung in Form von lapidaren »Vier Thesen« abschließen, auf deren Abdruck er jedoch in der Erstausgabe verzichtete. ${ }^{4}$ Zum anderen entwarf er auf siebenundzwanzig fieberhaft skizzierten Bögen einen "Schluß«, der die Beziehungen zwischen "Bild und Erfahrung" in theoretischen Begriffen zu definieren versuchte. ${ }^{5}$ Schließlich und vor allem aber hatte er sich im Zuge der Abfassung der Arbeit über Botticelli von 1888 an darum bemüht, das Ganze philosophisch auszuarbeiten; Skizzen dazu finden sich in einem voluminösen Manuskript mit dem ehrgeizigen Titel Grundlegende Bruchstücke zu einer monistischen Kunstpsychologie. ${ }^{6}$ All dies zeigt, mit wie viel Bedeutung die fünfzehn Zeilen der "Vorbemerkung» in den Augen des jungen Warburg aufgeladen waren.

3 Im Rückblick fällt es nicht allzu schwer, in den drei kurzen Absätzen, aus denen dieser Text besteht, die drei Hauptbegriffe wiederzuerkennen, die auf lange Sicht die Grundlage der Warburg'schen Kunstgeschichte bilden sollten. Der erste Absatz besagt, dass die Suche nach den - philosophischen oder poetischen - "Quellen« Botticellis darauf abzielt, »das, was die Künstler des Quattrocento an der Antike sinteressirteく, klarzulegen «. ${ }^{7}$ Jenseits der immer noch vagen Vorstellungen des »Interesses«, ja auch des »Einflusses«, ging es darum, Schritt für Schritt den Begriff des »Nachlebens« als zeitliches Modell $\mathrm{zu}$ etablieren, das den von Warburg beobachteten geschichtlichen Paradoxa und »Quellenbewegungen« gerecht zu werden vermag.

4 Der zweite Absatz isolierte, mit derselben Nüchternheit, ein bevorzugtes visuelles >Vehikel dieser zeitlichen Prozesse. Eben darauf konzentriert sich die ganze These visuell: »Es lässt sich nämlich hierbei Schritt für Schritt verfolgen, wie die Künstler und deren Berather in >der Antike` ein gesteigerte äussere Bewegung verlangendes Vorbild sahen und sich an antike Vorbilder anlehnten, wenn es sich um Darstellung äusserlich bewegten Beiwerks - der Gewandung und der Haare - handelte. ${ }^{8}$

5 Die gesteigerte - intensivierte - Bewegung als formales Werkzeug einer Antikenerinnerung? Warburg nahm hier natürlich bereits vorweg, was er einige Jahre später als »Pathosformeln« bezeichnen sollte. Die Wirksamkeit dieser figurativen Mittel ließ sich in den Augen des jungen Kunsthistorikers nämlich nur begreifen, indem man eine richtiggehende Psychologie - ja Metapsychologie - des Bildes ins Spiel brachte, die in der Lage wäre, seine formale und anthropologische Notwendigkeit zum Ausdruck zu bringen. Der dritte Absatz beruft sich dazu auf einen dritten Begriff, der derart abrupt eingeführt wird, dass er immer noch ziemlich rätselhaft erscheint; es handelt sich um den Begriff der »Einfühlung« [franz. empathie]:

»[...] dass dieser Nachweis für die psychologische Aesthetik deshalb bemerkenswerth ist, weil man hier in den Kreisen der schaffenden Künstler den Sinn für den ästhetischen Akt der `Einfühlung in seinem Werden als stilbildende Macht beobachten kann. ${ }^{9}$ 
Wie die drei Grazien in Botticellis Frühling bilden diese drei Grundbegriffe - Nachleben, Pathosformel, Einfühlung - einen unauflöslichen Kreis, der Warburgs gesamte Analyse durchzieht. Man kann ihre Dynamik verfolgen, deren bewundernswerte, berührende und bewegende Kraftlinien Botticellis Kunst (sowie die des Florentiner Quattrocento ganz allgemein) zum Ausdruck bringt. Man könnte auch verfolgen, wie sie im gesamten Werk des Kunsthistorikers ein beharrliches Motiv - das Leitmotiv oder Leitfossil ${ }^{10}$, wie Warburg gerne sagte - bilden, bis hin zum Gipfelpunkt, den unbestreitbar der Bilderatlas Mnemosyne darstellt.

Mit seiner verblüffenden Kunst der Montage sprengt Warburgs Bilderatlas den Rahmen der positivistischen Kunstgeschichte. Er zerschlägt die selbstproklamierten Gewissheiten sowohl der Wissenschaft, die sich ihrer Wahrheiten, als auch der Kunst, die sich ihrer Kriterien sicher ist. Er erfindet zwischen all dem liegende Bereiche forschenden Erkundens, heuristische Intervalle. Von definitiven Axiomen will er nichts wissen. Denn er beruht auf einer Erkenntnistheorie, die sich dem Risiko des Sinnlichen, und auf einer Ästhetik, die sich dem Risiko der Disparität aussetzt. In seiner überbordenden Fülle dekonstruiert dieser Bilderatlas die Ideale der Einmaligkeit, der Eigentümlichkeit, der Reinheit, der vollständigen Erkenntnis. Er ist kein Werkzeug zur logischen Ausschöpfung der gegebenen Möglichkeiten, sondern eines der unerschöpflichen Offenheit für noch nicht gegebene Möglichkeiten. Sein Prinzip, sein Antrieb ist nichts anderes als die Einbildungskraft. Einbildungskraft: ein wahrlich gefährliches Wort (wie auch bereits die Wörter Bild, Pathos oder Empathie bzw. Einfühlung). Wir sollten uns jedoch gemeinsam mit Goethe, Baudelaire oder Walter Benjamin ${ }^{11}$ in Erinnerung rufen, dass die Einbildungskraft, so verwirrend sie auch sein mag, nichts mit einer willkürlichen privaten Phantasie zu tun hat. Im Gegenteil, sie schenkt uns eine traverse - mit Querverbindungen arbeitende - Erkenntnis, und zwar durch ihr essentielles Montage-Vermögen, mit dem sie Verbindungen entdecken lässt gerade da, wo sie durch offenkundige Ähnlichkeiten insinuierte Verbindungen zurückweist -, die die unmittelbare Betrachtung nicht zu erkennen vermag.

7 Anthropologisch gesehen ist die Einbildungskraft zunächst einmal dasjenige, was uns befähigt, zwischen den entferntesten, heterogensten, inkommensurabelsten Wirklichkeitsordnungen Brücken $\mathrm{zu}$ schlagen. Walter Benjamin hat Warburgs Montagen aus dem Mnemosyne-Atlas vermutlich nicht gekannt, doch hat er eine exakte Beschreibung seiner grundlegenden Triebfedern geliefert, als er in seinem Aufsatz "Über das mimetische Vermögen" - eine beiden Denkern ganz offensichtlich gemeinsame Problematik - aus dem Jahre 1933 jenes "Lesen vor aller Sprache« erwähnte, das in der Fähigkeit bestehe, "'was nie geschrieben wurde, [zu] lesen«", und auch präzisierte, wo sich dies vollzieht: "aus den Eingeweiden, den Sternen oder Tänzen «. ${ }^{12}$ Die Tänze, sowie menschliche Gebärden ganz allgemein, sind das Wesentliche, bilden das Zentrum der Warburg'schen Sammlung, die von Anfang an als ein Bilderatlas von »Pathosformeln« gedacht war, jener Grundgesten, die von der Antike bis in unsere Zeit übermittelt - und transformiert - wurden: Gebärden der Liebe und des Kampfes, Gebärden des Triumphs und der Unterwerfung, der Erhöhung und des Falls, der Hysterie und der Melancholie, der Anmut und der Hässlichkeit, des lebhaften Begehrens und der Schreckensstarre ... 
Abb. 1

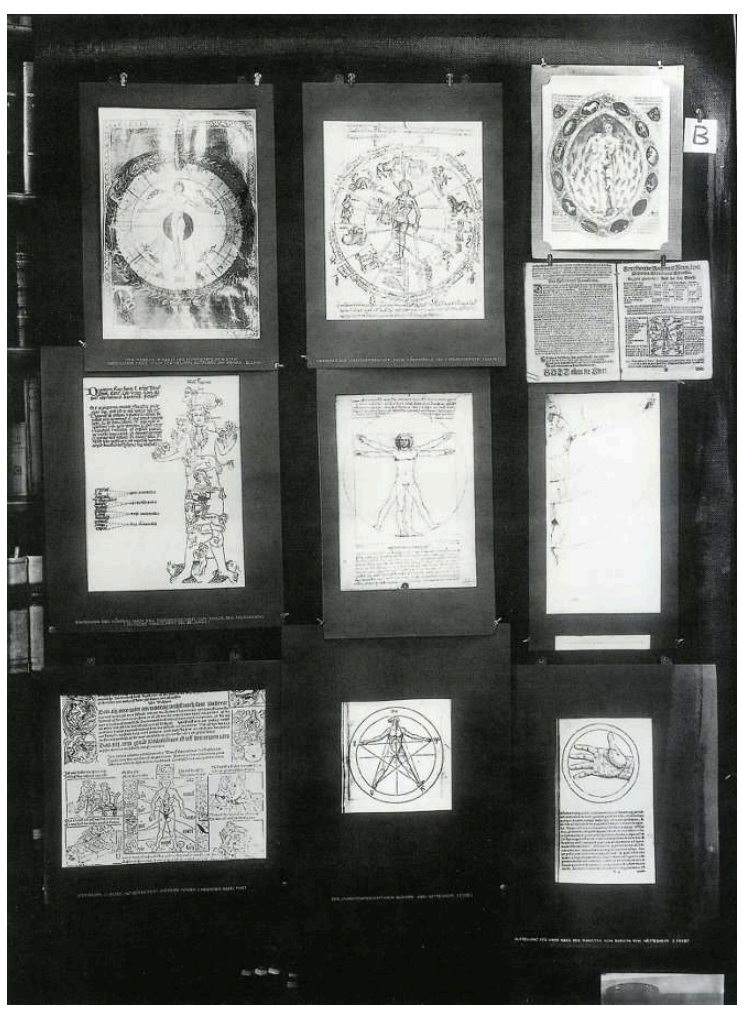

Aby Warburg, Bilderatlas Mnemosyne, 1927-1929. Bildtafel B. London, Warburg Institute Archive. Photo: The Warburg Institute.

Im Zentrum des Mnemosyne-Atlas steht also der Mensch, mit aller kontrastreichen Energie seiner Gedanken, Gebärden und Leidenschaften. Warburg hat jedoch Sorge getragen, dass diese Energie vor einem Hintergrund erscheint, der auf ihre konflikthafte Grenze hinweist, das Ungedachte, den Bereich des Nichtwissens: auf der einen Seite astra, auf der anderen monstra. Auf der einen Seite agiert der Mensch unter einem unendlichen Himmel, über den er sehr wenig weiß, daher sind die einleitenden Bildtafeln der Korrespondenz zwischen den Sternen und dem Menschen, das heißt der "Abtragung des kosmischen Systems auf den Menschen ${ }^{13}$ gewidmet (Abb.1). Auf der anderen Seite finden sich, symmetrisch dazu, die Abgründe der Welt der Eingeweide, wobei der Mensch auf Erden agiert, ohne genau zu verstehen, was ihn von innen heraus bewegt: seine eigenen »Monster«. Und der Bilderatlas suggeriert, dass es keine menschliche Geste ohne psychische Umwandlung gibt, keine Umwandlung ohne organische Flüssigkeiten, und keine organische Flüssigkeit ohne verborgene [secrète] Innerei, die sie sekretiert. 
Abb. 2

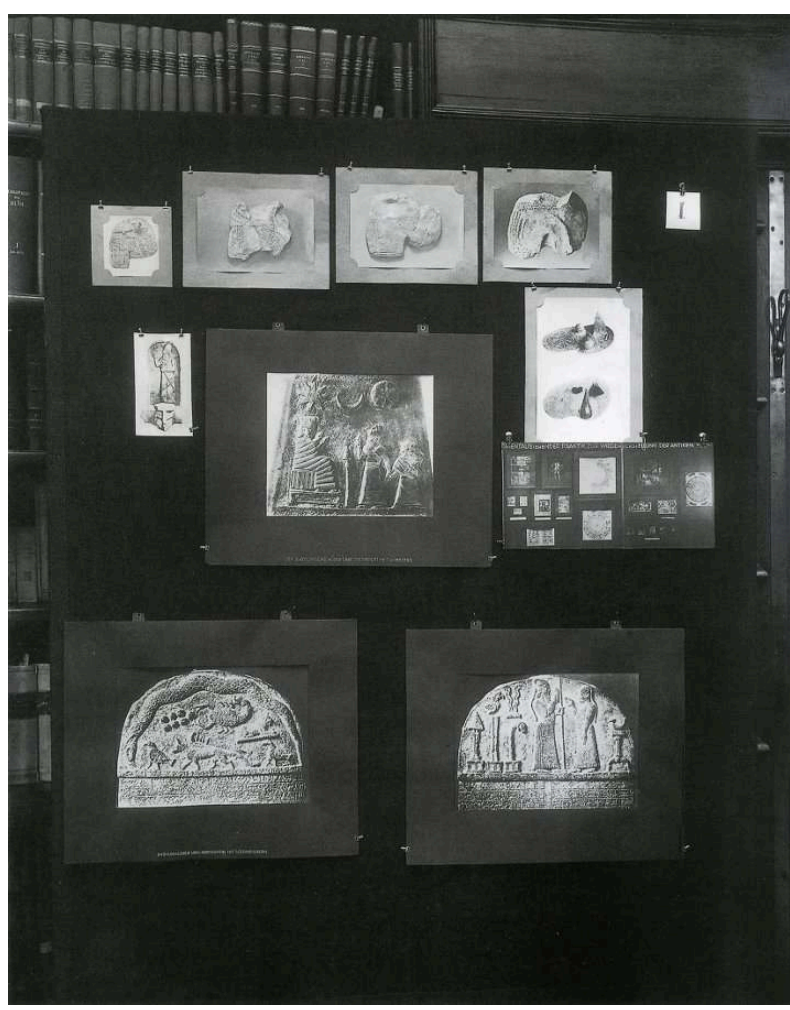

Aby Warburg, Bilderatlas Mnemosyne, 1927-1929. Bildtafel 1. London, Warburg Institute Archive.

Photo: The Warburg Institute.

9 Die Bildtafel 1 des Mnemosyne-Atlas ist in dieser Hinsicht so erstaunlich wie signifikant (Abb.2). Sie ist erstaunlich, weil sich neben leicht identifizierbaren Bildern wie den astronomischen oder astrologischen Figuren von Sonne, Mond oder Skorpion, sowie Königsfiguren (links ist Assurbanipal zu sehen), die möglicherweise auf den politischen Horizont oder zumindest politischen Gebrauch jedweder Weltvorstellung hindeuten, weil sich also neben all dem, an die Oberkante der Tafel gerückt, fünf brutale Tatsachen finden, fünf formlose Formen, die der westliche Kunsthistoriker vermutlich nur mit einiger Mühe wiederkennen wird. Man muss etwas näher hinsehen (Abb.3). Dann erfährt man - wenn man gleichzeitig geduldig bestimmte Bereiche der außerordentlichen Bibliothek erkundet, die Warburg zusammengestellt hatte ${ }^{14}$, den "Denkraum", von dem nichts, was er je unternahm, getrennt werden kann -, dass es sich um alte babylonische oder etruskische Darstellungen von Schafslebern handelt. 
Abb. 3

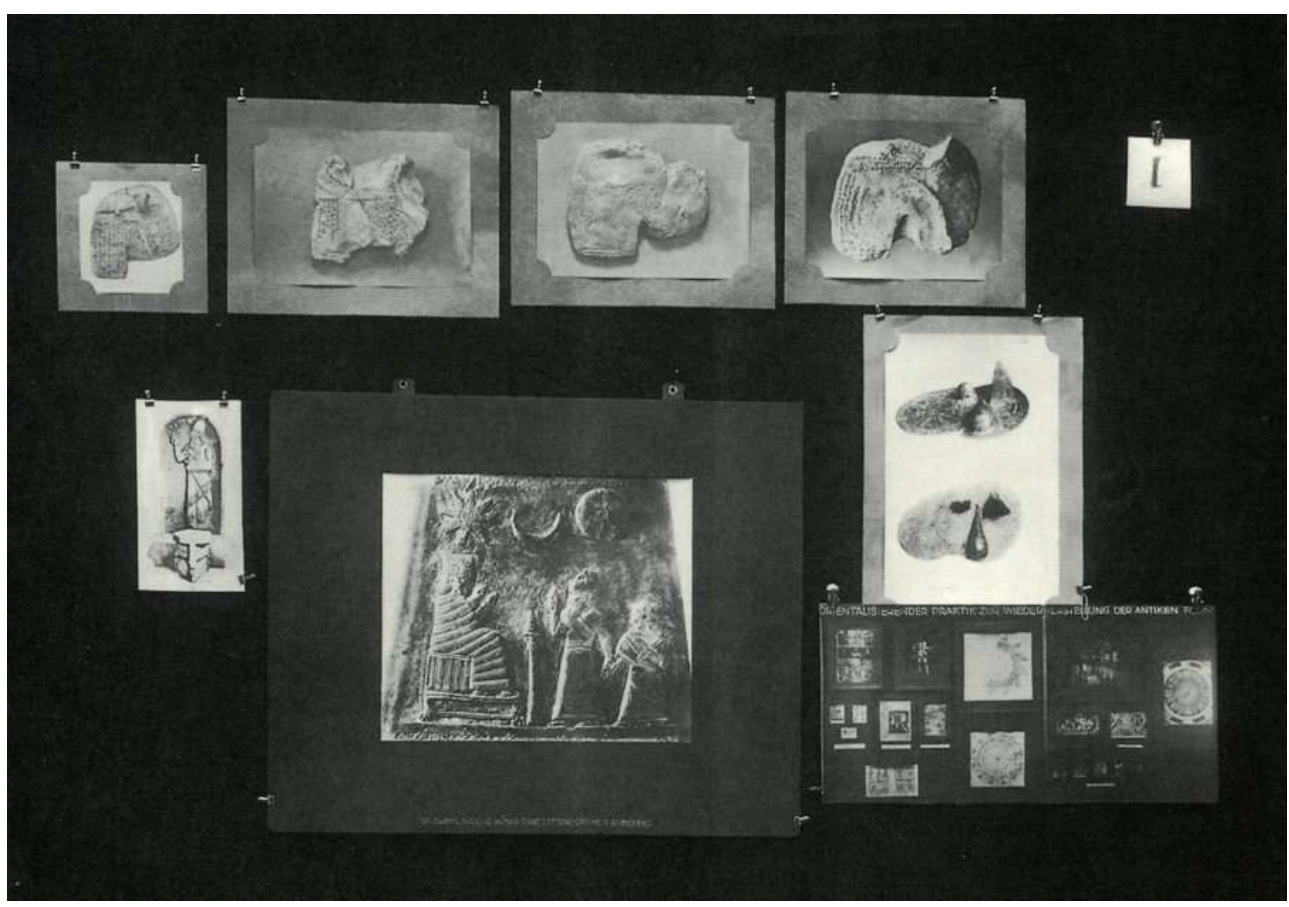

Aby Warburg, Bilderatlas Mnemosyne, 1927-1929. Bildtafel 1 (Detail). London, Warburg Institute Archive.

Photo The Warburg Institute.

10 Wie befremdlich! Wenn der Mnemosyne-Atlas als eine Schatzkammer des visuellen Wissens, als das Erbe unserer Zeit erscheint, müsste man nun anerkennen, dass das Initial-, ja Initiationsobjekt dieses Erbes - eines wertvollen Erbes, denn es geht hier um nichts Geringeres als unsere Kunstgeschichte in ihrer longue durée - dort in ein paar Schafslebern zu finden wäre, die sozusagen als die ersten »Sätze« einer Geschichte der abendländischen Kultur präsentiert werden! Das Verblüffende dieses Einstiegs, ganz oben auf Bildtafel 1 des Mnemosyne-Atlas, hat jedoch nichts Willkürliches an sich, und sei es nur deshalb, weil Warburg hier die dunklen Mächte der Einbildungskraft auf philosophischer und anthropologischer Ebene ernst nahm.

11 Vor allem aber sind diese formlosen Objekte, die der Bildhistoriker strategisch ausgewählt hatte, weder bedeutungslose noch einfache Gegenstände. Ihre Komplexität hängt gerade damit zusammen, dass sie als empathischeBilder, aber auch als dialektische Bilder fungieren: Bilder, die dazu bestimmt sind, diese heterogenen Räume, die die Verschlingungen der Eingeweide einerseits und die Himmelssphäre andererseits darstellen, zusammen zu montieren - und wechselseitig zu inkorporieren. Warburg hat einen beträchtlichen Teil seiner Forschungsarbeit Fragen der Astrologie gewidmet: Zeitliche Bewegungen in visuellen Konfigurationen $z u$ lesen - wie die Sternbilder [constellations] sie darstellen -, ist das nicht im Grunde ein elementares Paradigma aller Erkenntnis, die versucht, ausgehend vom Sinnlichen zu Intelligiblem zu gelangen? Und besteht darin, nebenbei bemerkt, nicht die hauptsächliche Arbeit eines jeden Archäologen oder Kunsthistorikers? Wie dem auch sei, Warburg hat sich lange darum bemüht, die kulturelle Bedeutung dieser astrologischen "Vorwissenschaft" - oder dieses astrologischen Vorauswissens um die Zukunft - für die ästhetische ${ }^{15}$ wie die politische und religiöse Geschichte ${ }^{16}$ der Renaissance zu erfassen. Auf der rechten Seite 
der bereits erwähnten Bildtafel hat Warburg übrigens zwei weitere Tafeln, die er für eine Ausstellung über die antike orientalische Astrologie konzipiert hatte ${ }^{17}$, in einer Art Medaillon als Bild im Bild aufgenommen. Als Inspiration dienten ihm damals die Arbeiten seines Freundes Franz Boll, dessen Ideen er seinen eigenen theoretischen Fragestellungen anpasste und dem er auch die berühmte Formel per monstra ad sphaeram entlieh. ${ }^{18}$

Die Schaulebern interessierten Warburg ${ }^{19}$ auch insofern, als sie in seinen Augen einen exemplarischen Fall jener historischen und geographischen Mobilität darstellten, deren bevorzugte Vehikel die Bilder sind: wandernde Bilder, die, wenn man sie zur Kenntnis nimmt, aus jedem "Kunststil« und aus jeder "Nationalkultur« - wie man fälschlicherweise sagt - eine essentiell gemischte, unreine, hybride Einheit machen. Eine Mischung oder Montage von heterogenen Dingen, Orten und Zeiten. Einer der entscheidendsten Beiträge der Warburg'schen Kunstgeschichte war seine Entdeckung, dass es selbst in jenen Errungenschaften des Abendlands, die am stärksten von "Klassik« und »Maß« geprägt sind - die griechisch-römische Kunst einerseits und die italienische Renaissance andererseits -, eine grundlegende Unreinheit gibt, die mit großen Wanderbewegungen verbunden ist, die nur eine Kulturwissenschaft ${ }^{20}$ zutage $^{2}$ fördern konnte, die dieses Namens würdig, das heißt in der Lage ist, in jeder visuellen Konfiguration die heterogenen räumlichen Bewegungen zu lesen. ${ }^{21}$ Wenn uns Warburg im weiteren Verlauf seines Bilderatlas Rembrandts Anatomien vor Augen führt, hat er sie daher zunächst von ihrer augenscheinlichsten - wissenschaftlichen, cartesianischen Bedeutung gelöst; dazu montierte er sie mit religiösen Szenen und Antikenerinnerungen, die uns diese Bilder im Sinne eines fernen Nachlebens der "magischen Anatomie «22 verstehen lassen, wodurch wir nicht zuletzt an die Schaulebern der ersten Bildtafel erinnert werden.

Die erste von diesen, oben links (Abb.3), ist eine babylonische Tonleber, die sich im British Museum befindet. Sie wurde vermutlich ca. 1700 vor Christus modelliert. Die drei anderen neben ihr stammen aus dem Vorderasiatischen Museum in Berlin und sind auf die erste Hälfte des 14. Jahrhunderts vor Christus datierbar. Es handelt sich hierbei um faszinierende und doppeldeutige Objekte, wie alle dialektischen Bilder es sind: In ihnen treffen mindestens zwei Zeitlichkeiten, zwei Welten, zwei Wirklichkeitsordnungen zusammen - das tiefste Innere und das fernste Äußere --, die normalerweise alles trennt. Einerseits handelt es sich um äußerst realistische Bilder: Die Schafsleber ist in Originalgröße dargestellt, wobei sie in etwa so erscheint, wie ein babylonischer "Hepatoskop« sie wohl hat sehen und handhaben können, der dem Körper des unmittelbar zuvor getöteten Tiers das blutige Organ entnahm und es auf einen Schautisch legte. Ein heutiger Anatom kann mühelos die gesamte Morphologie des Organs erkennen: die asymmetrischen Lappen, deren einer als lobus quadratus bezeichnet wird, den processus pyramidalis genannten Auswuchs, oben die Pfortader mit ihrer porta hepatis sowie die nach rechts abfallende Gallenblase. Die zahlreichen Tonmodelle, die Archäologen im Orient entdeckten, weisen sämtlich diese anatomische Präzision auf. ${ }^{23}$

Andererseits sind diese Objekte aber nicht nur einfache naturalistische Darstellungen. Das wird deutlich, sobald man bemerkt, dass die Leber aus dem British Museum - wie die anderen auch - mit Schriftzeichen bedeckt und in geometrische Zonen unterteilt ist, die von regelmäßigen Vertiefungen durchbrochen werden, die sich gleichsam strategisch über die gesamte Oberfläche verteilen. Die Schrift lässt an ein in Stein 
gemeißeltes Gesetz oder an einen eingravierten Spruch denken, die geometrische Aufteilung an ein geheimnisvolles Schachbrett. In einer grundlegenden Untersuchung der mesopotamischen Wahrsagepraktiken hat Jean Bottéro gezeigt, dass die sogenannte »deduktive Divination« einen beträchtlichen Bereich abdeckte, der von der schlichten Beobachtung von Naturerscheinungen - Gestirnen, Meteoriten, Sonnen- und Mondfinsternissen, Steinen, Pflanzen, Tieren sowie natürlich des Menschen selbst, der in seiner Physiognomie und sogar in seinen Träumen beobachtet wurde ${ }^{24}$ - bis zur komplexen Ausarbeitung künstlicher Situationen wie der Verteilung der Figuren bei einem Glücksspiel reichte ${ }^{25}$ (wie es auch heute noch geschieht, wenn Ihnen jemand »die Karten legt«, um die Zukunft vorherzusagen).

In der liturgischen Divination, zu der die Beschau der Leber von Opfertieren gehört, wird das Natürliche mit Künstlichem, die sinnliche Erkenntnis mit intelligibler Konstruktion überlagert. Daher sind in der Hepatoskopie empirische Präzision (die Nahsicht auf die Eingeweide) und wuchernde Symbolik ${ }^{26}$ (die Hellsichtigkeit, die von einer ganzen Dramaturgie von Fernbeziehungen zwischen den Göttern heimgesucht wird) aufs Engste vermischt. Die Tonlebern gleichen also Interfacesprédiction, Operatoren einer Transformation zwischen der aus der Nähe betrachteten Welt der Eingeweide und der von ferne angerufenen Sternenwelt. Anatomische Beobachtung und astrologische sowie magische Imagination sind untrennbar miteinander verbunden. ${ }^{27}$ So schreibt zum Beispiel Jean Nougayrol, eine babylonische Inschrift zitierend: "Der Sonnen-Gott fuhr fort, seinen Willen in den Bauch des Schafes einzuschreiben. Das Naturschauspiel war eine Botschaft, die gelesen werden konnte. «28 Die Innereien des Tierkörpers gaben also zu »lesen«, was auf der Himmelskarte und im Götterkorpus »nie geschrieben wurde«.

Die Rituale der Weissagung mittels Eingeweideschau stellen eine bizarre Mischung dar, wobei die Gesten, die der Körper gegenüber dem unförmigen blutigen Haufen des herausgerissenen Organs $\mathrm{zu}$ vollziehen hat, mit einem kasuistischen juridisch-religiösen Formalismus einhergingen, der von der Schrift beherrscht wurde. ${ }^{29}$ Der Einbildungskraft und dem Bild - das heißt dem Tonmodell selbst - oblag es, die so verschiedenen Wirklichkeiten $\mathrm{zu}$ "montieren $", \mathrm{zu}$ memorieren oder miteinander $\mathrm{zu}$ verbinden. Vor einer Götterstatue legten die Priester ein Tontäfelchen nieder, in welches die Frage eingraviert war, die ein hoher Würdenträger (in der Regel der König selbst, zum Beispiel Assurbanipal, sich an den Gott Schamasch wendend) dem Schicksal stellen wollte. Nachdem der Opferpriester das Opfertier geopfert und geöffnet hatte, wurde zunächst das Aussehen der Innereien und ihre Farbe sorgfältig betrachtet, dann trennte man die Leber vom übrigen Kadaver, wobei man die sie umgebenden Partien untersuchte, die als »Palast« der Leber bezeichnet wurden. Anschließend legte der Wahrsager oder bârû das noch warme Organ auf seine linke Hand oder auf einen Tisch und enthüllte all seine Besonderheiten. Nun wich das Messer dem Stilett, da der bârû auf der Grundlage überaus präziser Formelsammlungen einen ausführlichen Bericht verfasste, etwas von folgender Art:

Wenn ein "Standort« vorhanden, der »Pfad« zweifach, der linke auf dem rechten Pfad liegt: der Feind wird seine Waffen über die Waffen des Fürsten wüten lassen. Wenn KAL nicht vorhanden, ni-ip-hu, wenn auf der rechten Seite des Standortes ein »Finger« liegt: Fall des Heeres oder des Heiligtums. Wenn der linke Teil der Gallenblase festgewachsen: dein Fuß zertritt den Feind. Wenn der »Finger« und MÀš normal sind, wenn der rückwärtige Teil der Leber rechts verletzt ist: Verletzung des Kopfes, Sinnesänderung meines Heeres. Wenn das Untere "geht«, wenn SA.TI auf der Krone aufsitzt, der mittlere Leberfinger, seine Grundlage 
gelockert ist, wenn kaskasu hell ist, die irru sâhirûti XV [fünfzehn Darmverschlingungen], das Innere des Schafes normal.

Wenn der »Pfad« zweifach ist, der linke auf dem rechten Pfade liegt, wenn KAL nicht vorhanden, auf der rechten Seite des "Standortes« ein »Finger « liegt, wenn der rückwärtige Teil der Leber rechts verletzt ist, wenn SA.TI auf der Krone aufsitzt, 5 ungünstige (Zeichen) dabei, günstige Zeichen nicht vorhanden, es ist ungünstig. ${ }^{30}$ gestattete es, zu »lesen, was nie geschrieben wurde«. Rufen wir uns in Erinnerung, dass (be-)schauen [contempler] zunächst einmal heißt, eine Wirklichkeit der Natur dadurch zu betrachten, dass man sie als templum eingrenzt, das heißt als einen streng abgesteckten Bereich, in dem eine übernatürliche Wirkmacht Winke auf die Zukunft gibt, sodass das Betrachten des Raumes hier darauf hinausläuft, in die Zeit zu blicken. Von da ausgehend entwickelte sich aber eine ganze Dialektik der formlosen Materie, gesehen als eine Kartographie von Symptomen, die $\mathrm{zu}$ intensiven schriftlichen Interpretationsaktivitäten Anlass beziehungsweise ihnen eine Statt gaben [donnant lieu]. Der Tisch [table], auf dem die organische Lebermasse präsentiert wurde, wurde im Tonmodell zum Bild, das als Gedächtnisstütze und Orientierungshilfe diente, sowie anschließend zum Täfelchen [tablette], das Inschriften trägt, die sowohl diagnostischer Natur (indem sie penibel beschreiben) als auch prognostischer Natur sind (indem sie die jeder beobachteten Besonderheit innewohnenden "geheimen inneren Beziehungen der Dinge, die Entsprechungen und Analogien ${ }^{31}$ ins Unendliche hinein deklinieren).

Magisches Denken eben, wird man sagen. Man muss sich jedoch über den Inhalt eines solchen Ausdrucks verständigen. Der Religionssoziologe Stefan Czarnowski, der im Kreis um Émile Durkheim, Marcel Mauss und Henri Hubert wirkte, untersuchte den Begriff des divinatorischen templum unter dem Gesichtspunkt einer "Zerstückelung der Ausdehnung" und ihrer Eingrenzung in einem präzisen Rahmen, in dem alles gemäß einem neuen »System konkreter Qualitäten« transfiguriert wird, deren Interpretation nun so organisiert ist, dass sie den menschlichen Gesten, Handlungen und Entscheidungen als Orientierung dient. ${ }^{32}$ Jacques Vernant hat diese Analysen in einer klassischen Studie über die Psychologie der Divination fortgeführt, in der er die strukturelle Verwandlung beschrieb, die die Wahrnehmung des beobachteten Organs in der Technik der Opferschaupriester erfährt: »In dem Moment, da der Körper des Opfers geöffnet wird, wird er, ohne sein Aussehen zu ändern, durch eine Anrufung der für die verschiedenen Teile des Organismus zuständigen Gottheiten in einen Tempel sverwandeltı. Die Einflüsse der verschiedenen Gottheiten lokalisieren sich an der Stelle, die ihnen jeweils vorbehalten ist. «3 $^{33}$

Der Opferschaupriester sieht wohl [voit bien] - ja »(be-)schaut « [contemple] mit äußerster Aufmerksamkeit - die Tierleber, die vor ihm auf dem »Sektionstisch« liegt. Sein Tun besteht jedoch nicht nur darin, sie zu sehen und "gut« zu sehen [»bien« voir]: Er sieht sie anders. Die "Verwandlung", von der Jacques Vernant spricht, betrifft zuallererst eine entscheidende Modifikation im Status der Sichtbarkeit des betrachteten beziehungsweise beschauten Objekts [objet contemplé]: Es wird von einem sichtbaren Ding im empirischen Sinne des Wortes zum Träger für anderes, das zu ersehen oder vorherzusehen ist. $\mathrm{Zu}$ ersehen [à entrevoir], sagte ich: Das bedeutet nicht einfach "weniger gut sehen", sondern im Gegenteil: Sehen im Hinblick auf die "geheimen inneren Beziehungen der Dinge, die Entsprechungen und Analogien«. Es vollzieht sich 
eine strukturelle Verwandlung, weil hier, im überaus präzisen räumlichen und zeitlichen Rahmen des templum, das Ding als sichtbare Einheit einem System vielfältiger figuraler Relationen weicht, in dem alles Gesehene nur über Umwege, Beziehungen, Entsprechungen und Analogien gesehen wird.

Dazu muss jedoch der Raum selbst modifiziert werden: der Raum des Erscheinens, der Präsentation oder der Disposition des zu Sehenden. Er muss mit einem Tisch [table] ausgestattet werden, um diese Verwandlung des Blicks und der Bedeutung aufzunehmen, um das ganze Bündel figuraler Vielfältigkeiten zu versammeln, die darauf warten, gesehen zu werden. "Sobald ein Raum ausgerichtet, eingegrenzt und aufgeteilt ist - wobei diese Operationen keinen durch die aktuelle Situation implizierten Notwendigkeiten oder Bedürfnissen entsprechen, sondern einem Ritus folgen -, bekommt er eben dadurch einen symbolischen Wert, der ihn zu einem Einsatzfeld divinatorischer Praktiken werden lässt. « ${ }^{34}$ Das beginnt mit einer Reihe von präzisen, konkreten, technischen Handgriffen: sozusagen mit der Kunst, den Tisch "aufzustellen« oder $z u$ bereiten (Abb. 4). Und es endet mit der Etablierung einer neuen Erkenntnis, deren epistemologisches Profil Vernant abschließend skizziert: »Die Divination gründet hier also nicht auf einer affektiven Vermischung, sondern auf Klassifikationen, die ebenso konkret wie präzis sind, wenn auch nicht deckungsgleich mit unseren wissenschaftlichen Klassifikationen. $\ll^{35}$

Abb. 4

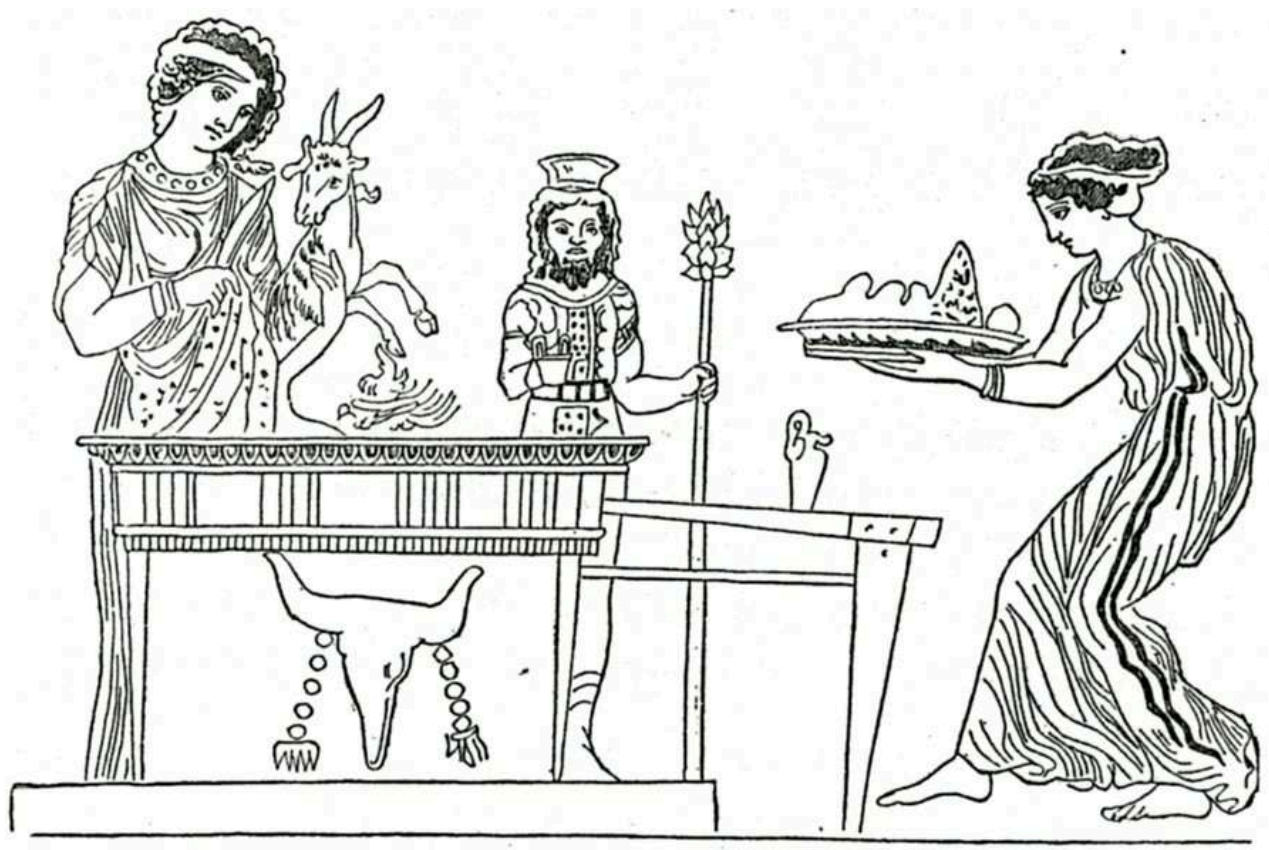

Opferszene im antiken Griechenland. Nach Daremberg, C. / Saglio, E. (Hg.) (1873): Dictionnaire des antiquités grecques et romaines, I, Paris, S. 349.

21 Jacques Vernant lädt uns in diesem Text dazu ein, den Begriff des "magischen « oder "mythischen Denkens« neu zu denken, der in den einseitigen - positivistischen oder neo-evolutionistischen - Theorien ganz von jener »Ideenvermischung", der verrückt spielenden Imagination, überschattet sein kann, die Platon und Cicero als erste im Namen der »Vernunft « oder der »Natur« gegeißelt hatten. Ein grundlegender Text in diesem Zusammenhang - aus dem Vernant möglicherweise für seine eigenen 
Hypothesen schöpfte - war der Artikel über »Einige primitive Formen von Klassifikation«, den Émile Durkheim und Marcel Mauss 1903 veröffentlicht hatten. Was uns im Zusammenhang mit Ritualen und Mythen von "primitivem Denken« sprechen lässt, beruht ihnen zufolge mitnichten auf »einfach[en] und elementar[en] « Funktionsweisen (wie damals gerade, nebenbei bemerkt, Freud für die Träume und die psychischen Symptome gezeigt hatte), sondern auf "geistige[n] Operationen, die [...] in Wirklichkeit überaus komplex sind «. ${ }^{36}$ Es ist also schlichtweg falsch, magisches Denken, wie zum Beispiel die Divination, nur unter dem Blickwinkel der empathischen Vermischung oder Ansteckung anzugehen, die aller begrifflichen Distinktion entgegengesetzt wäre. Beide wirken Hand in Hand, womit auch gesagt ist, dass es auf diesem Gebiet nicht hilfreich ist, Einbildungskraft und Vernunft mit aller Gewalt einander entgegenzusetzen.

Die Einbildungskraft ist genau an der Schnittstelle des Sinnlichen und des Intelligiblen angesiedelt (Kant hat bekanntlich versucht, sie als »verborgene Kunst in den Tiefen der menschlichen Seele« in eine Formel $\mathrm{zu}$ fassen, die er als "transzendentalen Schematismus « bezeichnet ${ }^{37}$ ). Nun sind aber in jeder Klassifikation, jeder Erkenntnis oder jeder technischen Praxis, so "primitiv« sie auch sein mögen, die sinnlich wahrnehmbaren Dinge und ihre intelligiblen Beziehungen gemeinsam am Werk. Marcel Mauss sollte behaupten, dass die »Partizipation« - das, was Warburg vom Begriff der Einfühlung* ausgehend in den Blick nahm - in ihrer strukturellen und operativen Kraft erkannt werden müsse, was sich durch die eingehende Untersuchung von Klassifikationen aus Australien, China, der Hopi oder der Winnebago verifizieren lasse. ${ }^{38}$ All dies beruht auf einer soziologischen und anthropologischen Grundintuition: Sowohl das affektive als auch das kognitive Element in "primitiven Klassifikationen", ihre monstra wie ihre astra, wären nichts anderes als die Fortsetzung einer bestimmten Organisation der Gesellschaft auf der Ebene der mentalen Vorstellungen und der intelligiblen Kategorien. ${ }^{39}$ 
Abb. 5
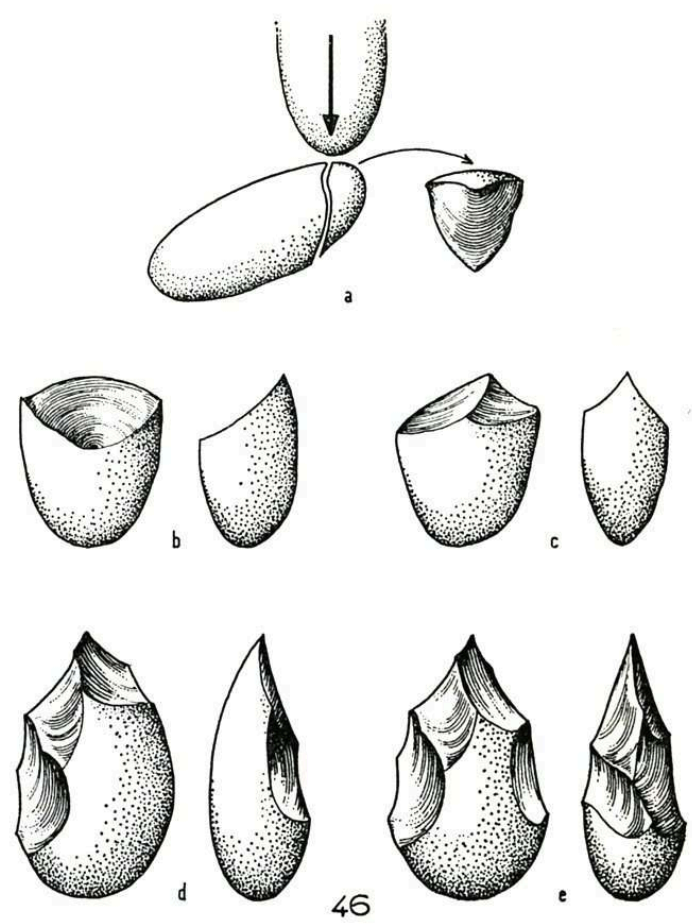

Prähistorische Technik der zerschlagenen Geröllsteine. Nach Leroi-Gourhan, A. (1980): Hand und Wort. Die Evolution von Technik, Sprache und Kunst, Frankfurt a.M.: Suhrkamp, S. 121 (Abb. 46).

Diesen soziologischen Blickwinkel gilt es jedoch durch den technischen Begriff der Operationskette zu ergänzen, den André Leroi-Gourhan in die Anthropologie einführte. Die Welt wird durch die technische Operation einerseits zerstückelt, wie an der prähistorischen "Geröllstein-Industrie« gut zu sehen ist, wo eine mit einem heftigen Schlag ausgeführte Zerlegung [démontage] roher Kieselsteine - eine Art Sezieren der Dinge, könnte man sagen - die ersten »distinkte[n] Form[en] « hervorbringt, wie LeroiGourhan sie nennt (Abb.5); andererseits betrachtet er das so erhaltene Werkzeug als eine richtiggehende "Sekretion des Körpers«, in der sich die beiden Bedeutungen des griechischen Wortes organon überschneiden. ${ }^{40}$ Es ist etwas schade, dass diese TechnikAnthropologie nicht tiefschürfender untersucht hat, was aus jeglichem Tisch[table] eine richtiggehende, Welt und Körper umfassende Apparatur macht, das heißt etwas viel Komplexeres als einen einfachen Träger. ${ }^{41}$ 

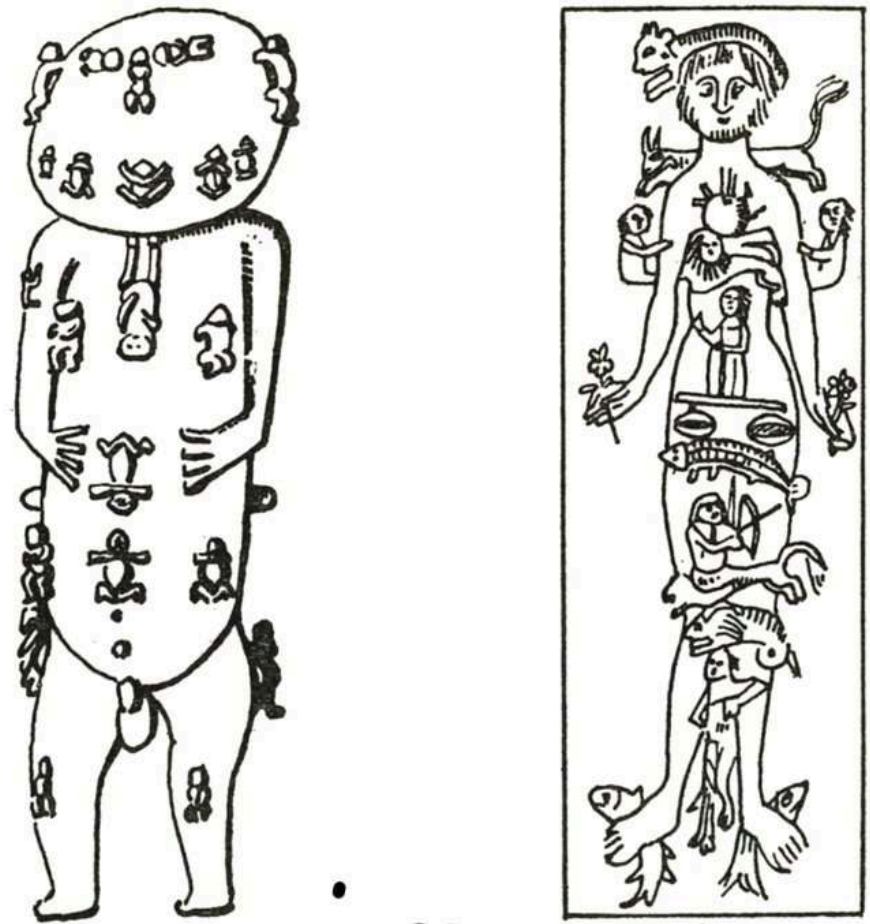

Polynesische Statuette (Tubai, 19. Jahrhundert) und Zodiakalmann (Frankreich, 16. Jahrhundert). Nach Leroi-Gourhan, A. (1980): Hand und Wort. Die Evolution von Technik, Sprache und Kunst, Frankfurt a.M.: Suhrkamp, S. 251 (Abb. 98).

In Hand und Wort findet sich jedoch ein entscheidendes, der Entstehung des Graphismus gewidmetes Kapitel, in dem Leroi-Gourhan bezeichnenderweise von prähistorischen Felsgravuren zu fernöstlichen Weihegaben sowie schließlich zu zwei figürlichen Darstellungen übergeht, die nebeneinander abgebildet werden, obwohl sie zwei völlig verschiedenen kulturellen Kontexten entspringen (Abb.6). Linker Hand ist dort die Zeichnung einer polynesischen Statuette zu sehen: Auf dem Körper des großen Gottes des Ozeans ist der Mythos von der Schöpfung der Götter und der Menschen dargestellt. Rechter Hand ist ein aus der Renaissance stammender Holzschnitt mit einem »Zodiakalmann" reproduziert, von dem Aby Warburg einige Varianten aus der Zeit zwischen dem 12. und dem 18. Jahrhundert auf die Tafel B seines Mnemosyne-Atlas setzte (Abb.1). In beiden Fällen wird der anthropomorphe Körper als ort der Zerstückelung und der Vielfältigkeit zugleich dargestellt [figuré]: Die Schwärme seltsamer Kreaturen, die ihn überziehen, scheinen seiner Oberfläche anzuhaften, aber auch seine körperliche Einheit zu zerreißen, insbesondere im Falle des Zodiakalmannes, der in seinem rechtwinkligen Rahmen auf einem Tisch zu liegen scheint, auf dem er seziert worden zu sein scheint, um das animalische - aber auch astrale - Gewimmel zutage zu fördern, das ihn entstellt [défigure].

Leroi-Gourhan hat in diesen graphischen Beispielen zurecht das gesehen, was er Operationsfelder nennt, wo der gezeichnete Strich, das Piktogramm, ja selbst der Buchstabe nicht zu trennen sind von den sie begleitenden technischen Gesten, den »rhythmisierten motorischen Bewegungen« oder den jeder Ritualisierung des Körpers inhärenten Elementen der Mündlichkeit. ${ }^{42}$ Claude Lévi-Strauss' strukturalistische 
Anthropologie mit ihrer "Logik der Klassifikationen " und ihren »Transformationssystemen ${ }^{43}$ würde bekanntlich des Fundaments entbehren - oder völlig abstrakt bleiben -, vergäße man die »Wissenschaft vom Konkreten«, die ihr zugrunde liegt. ${ }^{44}$ Das heißt jenes praktische Experimentieren, bei dem das »Basteln« bestimmter Dispositive - insbesondere visueller Art, wie die polynesische Statuette oder der Zodiakalmann aus der Renaissance - die notwendige Verbindung herstellt zwischen Körper und Denken, technischen Gesten und intelligiblen Kategorien, emphatischen Annäherungen und begrifflichen Distanzierungen, mythischen Erzählungen und wissenschaftlicher Erkenntnis ${ }^{45}$ (die Kritik, die Jack Goody seither an der "graphischen Vernunft" geübt hat, krankt meiner Ansicht nach an seiner Weigerung, den Graphismus im weiteren Kontext einer Verräumlichung des Körpers und des Denkens zu betrachten, wodurch zum Beispiel die Begriffe »Tisch « [table] und »Tafel« [tableau], die wir hier voneinander $\mathrm{zu}$ unterscheiden versuchen, einseitig aufeinander heruntergebrochen werden. ${ }^{46}$.

Wie die etruskischen oder babylonischen Schaulebern sind auch die polynesische Statuette und der Zodiakalmann, die Leroi-Gourhan ausgewählt hatte, organische Formen, die gleichzeitig die Funktion von Operationsfeldern übernehmen. Auf der Ausgangsfigur verteilen sich, gleichsam aufgepfropft, andere Figuren, die untereinander interagieren, mit ersterer jedoch inkommensurabel sind: Ein Riss in der Leber, der als »Palasttor« bezeichnet wird, eine anthropomorphe Darstellung, die von Tieren, Homunkuli und Fabelwesen nur so wimmelt. Was also ist, in diesem Kontext betrachtet, ein Operationsfeld? Ein bestimmter Ort - eingehegt als templum in jeglicher Ausdehnung, der Himmel, das Meer, ein flacher Stein, eine Schafsleber ... -, der die Fähigkeit besitzt, heterogene Wirklichkeitsordnungen einander begegnen zu lassen, und diese Begegnung anschließend zu einem Ort der Überdeterminierung zu machen. Ein "Tisch", auf den man bestimmte disparate Dinge zusammenzulegen beschließt, deren vielfältige "geheime innere Beziehungen« man $\mathrm{zu}$ erschließen versucht, ein Bereich mit eigenen Dispositions- und Transformationsregeln, um bestimmte Dinge, deren Verbindungen keineswegs evident sind, miteinander $\mathrm{zu}$ verbinden; und um diese Verbindungen, sobald sie einmal zutage gefördert wurden, zu Paradigmen einer Neulektüre der Welt zu machen.

Der theoretische Vorteil der von Aby Warburg und Leroi-Gourhan gewählten Beispiele besteht in ihrer Ausweitung dessen, was man vom Begriff des Operationsfelds spontan erwarten würde. Die Unordnung ist nur für denjenigen Unvernunft, der sich weigert, die Zerstückelung der Welt $\mathrm{zu}$ denken, $\mathrm{zu}$ respektieren und in gewisser Weise $\mathrm{zu}$ begleiten. Der Tisch [table] wäre also ein vorzüglicher Ort, um diese Zerstückelung (emphatisch) zu versammeln und (abstrakt) zu präsentieren. Um ihre grundlegende operative Bedeutung zu betonen, die in der - stets offenen - Möglichkeit besteht, sich zu wandeln und eine neue Konfiguration hervorzubringen. Auf diese Weise würde jeder Tisch auf seine Art die Teilung der Dinge sanktionieren: ihre Bestimmung, einverleibt, dann getrennt und schließlich neu verteilt zu werden. Daher die unmittelbar soziale, kulturelle und politische Dimension des Tisches: Die lateinische mensa bezeichnete zunächst eine Art Kuchen, der in vier Teile geteilt wurde, die man als Opfergaben für die Götter niederlegte, sowie als verzehrbare Nahrung auf einem Operationsfeld, das alsbald ihren Namen übernahm. ${ }^{47}$ In seiner Untersuchung zu den »heiligen Tischen « der Griechen und der Römer hat Christian Goudineau die Verbindung bemerkt, die zwischen den "Operationsfeldern" des Opferkults und den Gottheiten des Bodens und der Vegetation, vor allem Dionysos, besteht. ${ }^{48}$ Wie sollte man auch nicht darüber 
staunen, dass sämtliche Marmortische, sowohl die in religiösen Gebäuden als auch die zum häuslichen oder luxuriösen Gebrauch, aus einem Material gefertigt sind, das aus dem Inneren der Erde gewonnen wurde? ${ }^{49}$

Wie sollte man also daraus nicht ableiten, dass der Tisch vor allem als ein Operator der Umwandlung zwischen den Mächten der Natur und den Mächten der Kultur, den rohen Dingen und den organisierten Zeichen, der Zerstückelung der monstra und der Konstellation der astra dient? Ob es darum geht, auf ihm eine Mahlzeit einzunehmen, Opfergaben niederzulegen, einen Körper zu sezieren, Erkenntnisse zu organisieren, ein Gesellschaftsspiel zu spielen oder irgendeine magische Handlung vorzunehmen - in all diesen Fällen versammelt der Tisch Heterogenitäten und verleiht vielfältigen Beziehungen Form. Ich möchte nur daran erinnern, dass Goethe - dessen leidenschaftlicher Leser Warburg war - die Empathie bzw. Einfühlung schon frühzeitig problematisierte, als er, über eine einfache Symmetrie zwischen Objekt und Subjekt hinausgehend, von der Wirksamkeit eines Supplements sprach, das als Interface oder Umwandlungsoperator fungiert: "Alles, was im Subjekt ist, ist im Objekt und noch etwas mehr. Alles, was im Objekt ist, ist im Subjekt und noch etwas mehr. ${ }^{50}$ Der Tisch beziehungsweise die Tafel [table] - der Seziertisch, der Schautisch [table de divination], der Opfertisch, aber auch die Bildtafel [table d'atlas] - könnte diese Rolle des »noch etwas mehr« spielen.

\section{BIBLIOGRAPHIE}

Abusch, T. / Toorn, K. van der (Hg.) (1999): Mesopotamian Magic. Textual, Historical, and Interpretative Perspectives, Groningen: Styx Publications.

Baudelaire, C. (1975 [1857]): »Notes nouvelles sur Edgar Poe«, in: CEuvres complètes, hg. v. Cl.

Pichois, Bd. 2, Paris, S. 329; dt.: »Neue Anmerkungen zu Edgar Poe«, übers. v. E. Meister, in: ders., Sämtliche Werke/Briefe in acht Bänden, hg. v. F. Kemp u. Cl. Pichois, Bd. 2, München 1983, S. 341-362. Belting, H. (2008): Florenz und Bagdad. Eine westöstliche Geschichte des Blicks, München: C. H. Beck.

Benjamin, W. (1991 [1933]): „Über das mimetische Vermögen«, in: ders., Gesammelte Schriften, hg. v. R. Tiedemann u. H. Schweppenhäuser, Frankfurt a.M.: Suhrkamp, Bd. II.1, S. 210-213.

Bottéro, J. (1974): „Symptômes, signes, écritures en Mésopotamie ancienne«, in: Divination et Rationalité, hg. v. J.-P. Vernant, Paris: Seuil, S. 70-197.

Bottéro, J. (1997 [1987]): Mésopotamie: l'écriture, la raison et les dieux, Neuaufl., Paris: Gallimard.

Cohon, R. H. (1984): Greek and Roman Stone Table Supports with Decorative Reliefs, Ann Arbor: University Microfilms International.

Contenau, G. (1940): La divination chez les Assyriens et les Babyloniens, Paris: Payot.

Czarnowski, S. (1923): „Le morcellement de l'étendue et sa limitation dans la religion et la magie«, in: Actes du Congrès International d'histoire des religions tenu à Paris en octobre 1923, Paris: Librairie Honoré Champion, Bd. I, S. 339-359. 
Didi-Huberman, G. (2002a): L'Image survivante. Histoire de l'art et temps des fantômes selon Aby Warburg, Paris: Minuit; dt.: Didi-Huberman (2010 [2002]).

Didi-Huberman, G. (2002b): Ninfa moderna. Essai sur le drapé tombé, Paris: Gallimard; dt: DidiHuberman (2006 [2002]).

Didi-Huberman, G. (2006 [2002]: Ninfa moderna. Über den Fall des Faltenwurfs, übers. v. M. Ott, Zürich / Berlin: Diaphanes.

Didi-Huberman, G. (2010 [2002]): Das Nachleben der Bilder. Kunstgeschichte und Phantomzeit nach Aby Warburg, übers. v. M. Bischoff, Frankfurt a.M.: Suhrkamp.

Durkheim, E. / Mauss, M. (1974 [1903]): »De quelques formes primitives de classification. Contribution à l'étude des representations collectives«, in: Mauss, M. (1974): CEuvres, II. Représentations collectives et diversité des civilisations, hg. v. V. Karady, Paris: Minuit, S. 13-89; dt.: Durkheim, E. / Mauss, M.: »Über einige primitive Formen von Klassifikation. Ein Beitrag zur Erforschung der kollektiven Vorstellungen«, in: Durkheim, E. (1987): Schriften zur Soziologie der Erkenntnis, übers. v. M. Bischoff, Frankfurt a.M.: Suhrkamp, S. 169-256.

Ernout, A. / Meillet, A. (1959 [1932]): Dictionnaire étymologique de la langue latine, durchgesehene Neuaufl., Paris: Klincksieck.

Gadd, C. J. (1966): »Some Babylonian Divinatory Methods and their Interrelations«; in: $L a$ Divination en Mésopotamie ancienne et dans les régions voisines. XIV ${ }^{e}$ Rencontre assyriologique international, Paris: PUF, S. 21-34.

Goethe, J. W. von (1998 [1809-1810]): »Maximen und Reflexionen«, in: Goethes Werke. Hamburger Ausgabe in 14 Bänden, hg. v. E. Truntz, München: C. H. Beck, 12. Bd.

Goody, J. (1979 [1977]): La Raison graphique. La domestication de la pensée sauvage, ins Franz. übers. v. J. Bazin u. A. Bensa, Paris: Minuit.

Goudineau, C. (1967): „Iéraï trapézaï", Mélanges d'archéologie et d'histoire de l'École francaise de Rome, LXXIX, 1967, S. 77-134.

Jastrow, M. (1908): »Hepatoscopy and Astrology in Babylonia and Assyria«, in: Proceedings of the American Philosophical Society, XLVII (190), S. 646-676.

Kant, I. (1997 [1781-1787]): Kritik der reinen Vernunft, in: Werkausgabe in zwölf Bänden, hg. v. Wilhelm Weischedel, Bd. III, Frankfurt a.M.: Suhrkamp.,

Klauber, E. G. (1913): Politisch-Religiöse Texte aus der Sargonidenzeit, hg. v. Ernst Georg Klauber, Leipzig: Verlag von Eduard Pfeiffer.

Leiderer, R. (1990): Anatomie der Schafsleber im babylonischen Leberorakel. Eine makroskopischanalytische Studie, München et al.: Zuckschwerdt.

Leroi-Gourhan, A. (1971 [1943]): Évolution et techniques, I. L'homme et la matière, Neuaufl., Paris: Albin Michel.

Leroi-Gourhan, A. (1973 [1945]): Évolution et techniques, II. Milieu et techniques, Neuaufl., Paris: Albin Michel.

Leroi-Gourhan, A. (1974 [1964]: Le Geste et la parole, I. Technique et langage, Neuaufl., Paris: Albin Michel; dt.: ders. Hand und Wort. Die Evolution von Technik, Sprache und Kunst, Erster Teil: »Technik und Sprache«, übers. v. M. Bischoff, Frankfurt a.M.: Suhrkamp, S. 11-270. 
Leroi-Gourhan, A. (1974 [1965]): Le Geste et la parole, II. La mémoire et les rythmes, Neuaufl., Paris: Albin Michel; dt.: ders. 1980 [1974]): Hand und Wort, Zweiter Teil: »Das Gedächtnis und die Rhythmen«, übers. v. M. Bischoff, Frankfurt a.M.: Suhrkamp, S. 271-498.

Lévi-Strauss C. (1962): La Pensée sauvage, Paris: Plon; dt. ders. (1968 [1962]): Das wilde Denken, übers. v. H. Naumann, Frankfurt a.M.: Suhrkamp.

Mani, N. (1959-1967): Die historischen Grundlagen der Leberforschung, Basel: B. Schwabe.

Mauss, M. (1974 [1907a]): »Noms propres et classifications chez les Hopi«, in: ders. (1974), S. 9496.

Mauss, M. (1974 [1907b]): »Le problème des classifications en Afrique occidentale«, in: ders. (1974), S. 96-99.

Mauss, M. (1974 [1913]): »Divisions sociales et classifications chez les Omaha«, in: ders. (1974), S. $100-103$

Mauss, M. (1974 [1923]): »Mentalité primitive et participation«, in: ders. (1974), S. 125-131.

Mauss, M. (1974 [1925]): »Cultes, rituel et classifications chez les Winnebago«, in: ders. (1974), S. 103-105.

Mauss, M., (1974): Cuvres, II. Représentations collectives et diversité des civilisations, hg. v. V. Karady, Paris: Minuit.

Meyer, J.-W. (1983): »Lebermodelle«, in: Reallexikon der Assyrologie und Vorderasiatischen Archäologie, Bd. 6, hg. v. D. O. Edzard, Berlin / New York: Walter de Gruyter, S. 522-527.

Moss, C. F. (1988): Roman Marble Tables, Ann Arbor : University Microfilms International.

Nougayrol, J. (1966): »Trente ans de recherches sur la divination babylonienne (1935-1965)«, in: La Divination en Mésopotamie ancienne et dans les régions voisines. XIVe Rencontre assyrologique internationale, Paris: PUF, S. 6-19.

Nougayrol, J. (1968a): »La divination babylonienne«, in: La Divination, hg. v. A. Caquot u. M. Leibovici, Paris: PUF, Bd. I, S. 25-81.

Nougayrol, J. (1968b): »Le foie >d'orientation〈 BM 50494«, Revue d'assyrologie et d'archéologie orientale, LXII, S. 31-50.

Plinius der Ältere, Naturalis Historia XXXVI (deutsch: Naturkunde, lat.-dt., hg. v. R. König u. G. Winkler, Bd. 36: Buch XXXVI: Die Steine, hg. u. übers. v. R. König in Zusammenarbeit mit J. Hopp, Darmstadt: Wissenschaftliche Buchgesellschaft 1992).

Reiner, E. (1995): Astral Magic in Babylonia, Philadelphia: The American Philosophical Society.

Rutten, M. (1938): »Trente-deux modèles de foies en argile inscrit provenant de Tell-Hariri (Mari)«, Revue d'assyrologie et d'archéologie orientale, XXXV, S. 36-70.

Vernant, J. (1948): »La divination. Contexte et sens psychologique des rites et des doctrines«, Journal de psychologie normale et pathologique, VI, S. 299-325.

Warburg, A. (1888-1905): Grundlegende Bruchstücke zu einer monistischen Kunstpsychologie, London, Warburg Institute Archive, III, 43.1-2.

Warburg, A. (1892): Schluß, London, Warburg Institute Archiv, III.38.4, 1.

Warburg, A. (2010 [1893]): »Sandro Botticellis >Geburt der Venus` und >Frühling«. Eine Untersuchung über die Vorstellungen von der Antike in der italienischen Frührenaissance«, in: Warburg (2010), S. 39-123. 
Warburg, A. (2010 [1912]): »Italienische Kunst und internationale Astrologie im Palazzo Schifanoja zu Ferrara«, in: Warburg (2010), S. 373-400.

Warburg, A. (2010 [1913]): „Die Fixsternhimmelsbilder der Sphaera Barbarica auf der Wanderung von Ost nach West«, in: Warburg (2010), S. 326-348.

Warburg, A. (2010 [1920]): »Heidnisch-antike Weissagung in Wort und Bild zu Luthers Zeiten«, in: Warburg (2010), S. 424-491.

Warburg, A. (1925a): Franz Boll zum Gedächtnis, London, Warburg Institute Archiv, III.94.1 [Vgl. ders., "Die Einwirkung der Sphaera barbarica auf die kosmischen Orientierungsversuche des Abendlandes« (Vortrag in Gedenken an Franz Boll, 25. April 1925), in: ders., »Per Monstra ad Sphaeram. Sternglaube und Bilddeutung. Vortrag in Gedenken an Franz Boll und andere Schriften 1923-1925, hg. v. D. Stimilli unter Mitarbeit v. Cl. Wedepohl, München / Hamburg: Dölling und Galitz 2008, S. 63-127. Kleine Schriften des Warburg Institute London und des Warburg Archivs im Warburg Haus Hamburg, Heft 3 (Es handelt sich hierbei um eine Ausgabe nach WIA III.94.2.1 »der von Warburg vorgetragene Text und das Handexemplar, das er in Hinsicht auf die Veröffentlichung überarbeitet hat« -, vgl. dazu »Anmerkung zum Text«, ebd., S. 138 ff.); A.d.Ü.].

Warburg, A. (1925b). Hepatoscopia, London, Warburg Institute Archiv, III, 132.3.

Warburg, A. (1998 [1926]): »Orientalisierende Astrologie«, in: ders., Gesammelte Schriften I. Die Erneuerung der heidnischen Antike. Kulturwissenschaftliche Beiträge zur Geschichte der europäischen Renaissance, hg. v. G. Bing u. F. Rougemont, Leipzig / Berlin: Teubner 1932; neu hg. v. H. Bredekamp u. M. Diers, Berlin: Akademie Verlag, Bd. I.2, S. 559-565.

Warburg, A. (2003 [1927-1929]): Der Bilderatlas Mnemosyne, in: Gesammelte Schriften, Bd. II.1, 2., durchgesehene Auflage, hg. v. M. Warnke u. C. Brink, Berlin: Akademie Verlag.

Warburg, A. (2010): Werke in einem Band, auf der Grundlage der Manuskripte und Handexemplare hg. und kommentiert v. M. Treml, S. Weigel u. P. Ladwig, Berlin: Suhrkamp.

Wind, E. (1979 [1931]): »Warburgs Begriff der Kulturwissenschaft und seine Bedeutung für die Ästhetik«, in: Aby M. Warburg, Ausgewählte Schriften und Würdigungen, hg. v. D. Wuttke, Baden-Baden: Valentin Koerner, S. 401-417.

Wölfflin, H. (1886): Prolegomena zu einer Psychologie der Architektur, München: Wolf \& Sohn.

\section{NOTES}

1. Vgl. Wölfflin (1886).

2. Warburg (2010 [1893]), S. 39 f. (Die Druckfahnen mit und ohne die Vorbemerkung befinden sich in London, Warburg Institute Archive, III.39.6, 1-5).

3. Warburg (2010 [1893]), S. 57.

4. Warburg (2010 [1893]), S. $108 \mathrm{f}$.

5. Warburg (1892).

6. Warburg (1888-1905).

7. Warburg (2010 [1893]), S. 39.

8. Warburg (2010 [1893]), S. 39.

9. Warburg (2010 [1893]), S. $39 \mathrm{f}$.

10. [Im Orig. deutsch; vgl. Warburg (2010 [1913]), S. 331; A.d.Ü.]

11. Vgl. Didi-Huberman (2002a), S. 391-505; dt.: Didi-Huberman (2010 [2002]), S. 431-559; DidiHuberman (2002b), S. 127-141; dt.: Didi-Huberman. (2006 [2002]), S. 139-157.

12. Benjamin (1991 [1933]), S. 213. 
13. Warburg (2003 [1927-1929]), S. 10.

14. Insbesondere unter den Siglen FEI, FME und FMH.

15. Warburg (2010 [1912]), S. 373-400.

16. Warburg (2010 [1920]), S. 424-491.

17. Warburg (1998 [1926]), S. 559-565.

18. Warburg (1925a), S. 2-5.

19. Vgl. Warburg (1925b).

20. [Im Orig. deutsch; A.d.Ü.]

21. Vgl. Wind (1979 [1931]).

22. Warburg, (2003 [1927-1929]), S. 124 f.

23. Vgl. Rutten (1938), S. 36-70; Contenau (1940), S. 235-283; Mani (1959-1967); Nougayrol (1968b), S. 31-50; Meyer (1983), S. 522-527; Leiderer (1990) (mit einem anatomischen Bildatlas).

24. Vgl. Bottéro (1974), S. 100-111.

25. Bottéro (1974), S. $123 \mathrm{f}$.

26. Bottéro (1974), S. 134-143 (Ritual), S. 143-168 (Empirismus) und S. 168-193 (Rationalisierung). Vgl. Gadd (1966), S. 21-34.

27. Vgl. Jastrow (1908), S. 646-676; Nougayrol (1966), S. 6-19; Nougayrol (1968a), S. 25-81; Reiner (1995); Abusch / van der Toorn (1999).

28. Nougayrol (1968a), S.32.

29. Vgl. Bottéro (1987), S.233-251.

30. Zitiert aus Contenau (1940), S. 262 [deutsch zitiert nach Klauber (1913), S. 104; Anführungsund Satzzeichen sowie Klammerergänzung nach der im Orig. zitierten franz. Übersetzung; A.d.Ü.].

31. Baudelaire (1975 [1857]), S. 329; dt.: Baudelaire (1983 [1857]), S. 352.

32. Czarnowski (1925).

33. Vernant (1948), S. 305

34. Vernant (1948), S. $311 \mathrm{f}$.

35. Vernant (1948), S. 311.

36. Vgl. Durkheim / Mauss (1974 [1903]), S. 13; dt.: Durkheim / Mauss (1987), S. 171.

37. Kant (1997 [1781-1787]), S. 190.

38. Vgl. Durkheim / Mauss (1974 [1903]), S. 19-81; dt.: Durkheim / Mauss (1987), S. 178-248; Mauss (1907a), S. 94-96; Mauss (1907b), S. 96-99; Mauss (1913), S. 100-103; Mauss (1923), S. 125131; Mauss (1925), S. 103-105.

39. Vgl. Durkheim / Mauss (1974 [1903]), S. 82-89; dt.: Durkheim / Mauss (1987), S. 171-178.

40. Leroi-Gourhan (1974 [1964]), S. 130-133; dt.: Leroi-Gourhan (1980 [1974]), S. 120-123. Vgl. Leroi-Gourhan (1971 [1943]), S. 47-64.

41. Vgl. Leroi-Gourhan (1973 [1945]), S. 183 u. 283.

42. Leroi-Gourhan (1974 [1964]), S. 261-300; dt.: Leroi-Gourhan (1980 [1974]), S. 237-270; LeroiGourhan (1974 [1965]), S. 26-34; dt.: Leroi-Gourhan (1980 [1974]), S. 288-295.

43. Vgl. Lévi-Strauss (1962), S. 48-143; dt.: Lévi-Strauss (1968 [1962]), S. 49-129.

44. Lévi-Strauss (1962), S. 3-47; dt.: Lévi-Strauss (1968 [1962]), S. 11-48.

45. Lévi-Strauss (1962), S. 33; dt.: Lévi-Strauss (1968 [1962]), S. 29 ff.

46. Goody (1979 [1977]), S. 110-111 (sowie insgesamt S. 108-139).

47. Ernout / Meillet (1959 [1932]), S. 397.

48. Goudineau (1967), S. 85-119.

49. Plinius der Ältere, Naturalis Historia XXXVI, I, 1-4; Cohon (1984); Moss (1988).

50. Goethe (1998 [1809-1810]), S. 436 (§ 515). 
INDEX

Mots-clés : empathie, Aby Warburg, foie divinatoire, participation

Schlüsselwörter : Empathie, Einfühlung, Aby Warburg, Schauleber, Partizipation

\section{AUTEURS}

\section{GEORGES DIDI-HUBERMAN}

Georges Didi-Huberman ist Dozent an der Ecole des hautes études en sciences sociales (Paris). Nähere Informationen finden Sie hier. 